\title{
\begin{tabular}{l|l} 
Mitraries & DSpace@MIT
\end{tabular}
}

\author{
MIT Open Access Articles
}

\section{High-throughput three-dimensional (3D) lithographic microfabrication in biomedical applications}

The MIT Faculty has made this article openly available. Please share how this access benefits you. Your story matters.

Citation: Kim, Daekeun, and Peter T. C. So. “High-throughput three-dimensional (3D) lithographic microfabrication in biomedical applications." Multiphoton Microscopy in the Biomedical Sciences X. Ed. Ammasi Periasamy, Peter T. C. So, \& Karsten Konig. San Francisco, California, USA: SPIE, 2010. 75691V-5. (C2010 SPIE--The International Society for Optical Engineering.

As Published: http://dx.doi.org/10.1117/12.843160

Publisher: Society of Photo-optical Instrumentation Engineers

Persistent URL: http://hdl.handle.net/1721.1/58557

Version: Final published version: final published article, as it appeared in a journal, conference proceedings, or other formally published context

Terms of Use: Article is made available in accordance with the publisher's policy and may be subject to US copyright law. Please refer to the publisher's site for terms of use. 


\title{
High-Throughput Three-Dimensional (3D) Lithographic Microfabrication in Biomedical Applications
}

\author{
Daekeun Kim ${ }^{\mathrm{a}}$ and Peter T. C. So ${ }^{\mathrm{a}, \mathrm{b}}$ \\ Department of Mechanical Engineering and Biological Engineering ${ }^{\mathrm{b}}$, Massachusetts Institute of \\ Technology, Cambridge, MA USA 02139-4301
}

\begin{abstract}
Two-photon excitation microfabrication has been shown to be useful in the field of photonics and biomedicine. It generates 3D microstructures and provides sub-diffraction fabrication resolution. Nevertheless, laser direct writing, the most popular two-photon fabrication technique, has slow fabrication speed, and its applications are limited to prototyping. In this proceeding, we propose high-throughput 3D lithographic microfabrication system based on depthresolved wide-field illumination and build several 3D microstructures with SU-8. Through these fabrications, 3D lithographic microfabrication has scalable function and high-throughput capability. It also has the potential for fabricating 3D microstructure in biomedical applications, such as intertwining channels in 3D microfluidic devices for biomedical analysis and 3D cell patterning in the tissue scaffolds.
\end{abstract}

Keywords: Two-photon absorption, lithography, SU-8, 3D fabrication

\section{INTRODUCTION}

Three-dimensional (3D) microfabrication techniques have been used extensively for prototype making. One popular method is two-photon excitation (TPE) microfabrication. The technology of TPE microfabrication [1-3] has been under development for over twenty years. Importantly, this microfabrication technique can utilize a variety of photointeractions to alter material properties in 3D. TPE microfabrication has found a broad range of applications in the fields such as 3D optical storage [4, 5], tissue scaffold [6, 7], photonic crystal structure [8, 9], and microfluidic devices [10, 11]. One of the limits of TPE microfabrication is its low throughput. TPE typically achieves 3D resolution by focusing ultrafast optical pulses to a diffraction-limited focus using a high numerical aperture (NA) lens. The nonlinear dependence of material responses to the excitation power distribution provides the depth discrimination. Since this fabrication processing is carried out on a point-by-point basis, known as laser direct writing, the throughput of fabrication is ultimately limited by the sequential nature of this method and the mechanical limit of the scanner. Although TPE microfabrication provides very attractive submicron lateral resolution and 3D optical sectioning, the use of the TPE approach is limited to laboratory investigations and prototype fabrication. Therefore, there is a need to improve the throughput of TPE microfabrication to enable commercial mass-production. We introduce 3D lithographic microfabrication technique based on depth-resolved wide-field illumination (DRWFI) $[12,13]$. It has found a number of applications in the nonlinear microscopy [14-17] and is also important for 3D microfabrication field since imaging and lithography has duality. During imaging, the sample is located at the object plane of the objective, and image is formed at image-conjugate plane. If the photomask is located at image-conjugate plane, the image of the photomask will be projected on the specimen, which is located in the objective plane of the objective.

In this proceeding, 3D-resolved lithographic microfabrication system is implemented, and its capabilities are demonstrated in terms of 3D microstructures built with SU-8 photopolymer. The potential for biomedical applications such as microfluidics and tissue engineering is also discussed.

\section{3D LITHOGRAPHIC MICROFABRICATION}

The schematic diagram of 3D lithographic microfabrication is shown in figure 1. Femtosecond pulsed laser is incident on reflective diffraction grating surface. It disperses light at different angles, depending on corresponding wavelength since optical pulse consists of a packet of wavelengths in spectral domain. After demagnifying lens pair, the optical

Multiphoton Microscopy in the Biomedical Sciences X, edited by Ammasi Periasamy, Peter T. C. So, Karsten König, Proc. of SPIE Vol. $7569,75691 \mathrm{~V} \cdot$ ( ) 2010 SPIE · CCC code: 1605-7422/10/\$18 - doi: 10.1117/12.843160 
pulse is restored where the image conjugate plane for specimen is located. By inserting optical mask, the image gets projected at the focal plane of the objective and the optical pulse is restored. Therefore, maximum photon flux occurs only at the focal plane with patterns formed by the optical mask and 2D microstructure is fabricated only at given depth. Photon flux rapidly decreases far from the focal plane, and this results in generating optical sectioning capability. By moving the objective in axial direction and changing optical mask, another 2D microstructure at different depth, and 3D microstructure are built by stacking up a series of $2 \mathrm{D}$ microstructures.

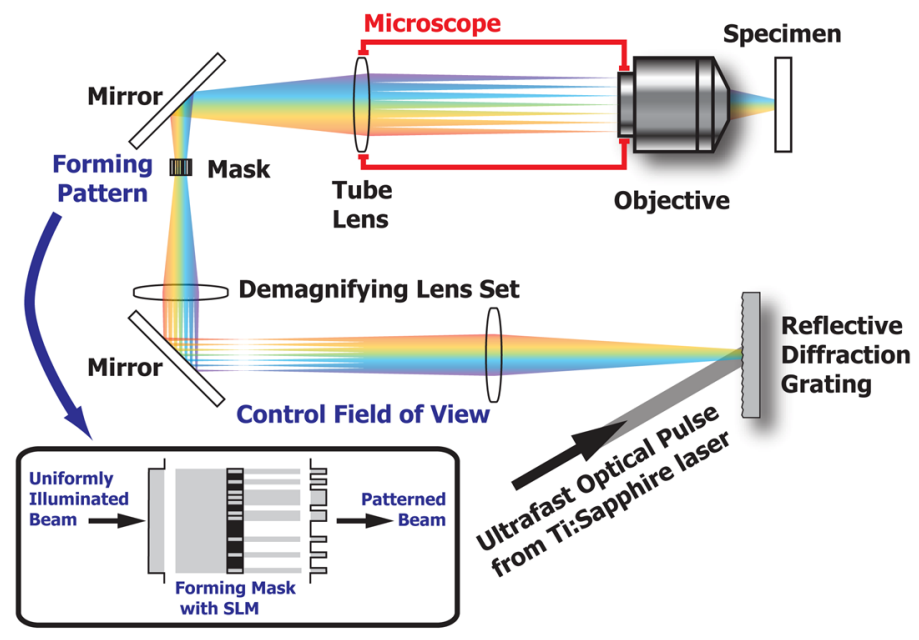

Figure 1. Schematic diagram of 3D lithographic microfabrication system

\section{MICROSTRUCTURES WITH SU-8 PHOTOPOLYMER}

In this section, several types of 3D microstructures are demonstrated in order to show its scale and high-throughput capability for 3D lithographic microfabrication. SU-8 was chosen as the photopolymer, since its polymerization is working very well with TPE [18]. Cover glass was used as a SU-8 substrate instead of silicon wafer commonly used in order to avoid back reflection on its surface. Since cover glass and SU-8 do not have strong adhesion, $2 \mu \mathrm{m}$ thick SU-8 layer was first cured on the cover glass. Then, we coated SU-8 thick enough to make the microstructures. All the processes except for the lithography are standard ones. For the lithography process, we used our own 3D lithographic microfabrication system. Figure 2 describes the protocol for SU-8 sample preparation in detail. For SU-8 microfabrication, Titanium Sapphire pulsed laser was used to facilitate two-photon excitation (pulse width is $\sim 100 \mathrm{fs}$ and pulse repetition rate is $80 \mathrm{MHz}$ ).

First, 2.5D microstructures were made with MIT logos. As seen in figure 3, roughly 1:5 high-aspect-ratio was achieved. Second, 3D structures similar to photonic crystal was generated by stacking up log piles in figure 4(a). In addition, the square with hole pattern was used as an optical mask, and overhanging structure was fabricated by overlapping two 3D structures in different depths. These 3D structure demonstrate that our microfabrication technique has 3D fabrication capability. To show the scalability, we made different scale structures with same photomask by using different magnification objectives. Although the optical resolution was sacrificed since lower magnification objective has lower NA, the structures were clearly scalable, as shown in figure 5. Lastly, we fabricated large area structure to demonstrate high-throughput capability by using fabrication mosaic. The size of each pattern is about $50 \mu \mathrm{m}$, and the total dimension is about $1 \mathrm{~mm}$ length by $1 \mathrm{~mm}$ width by $10 \mu \mathrm{m}$ height. The fabrication time for these structures is relatively short, compared with laser direct writing method. Currently, fabrication time is limited by pulse peak power; the use of regenerative amplifier may enable high-throughput mass production. 


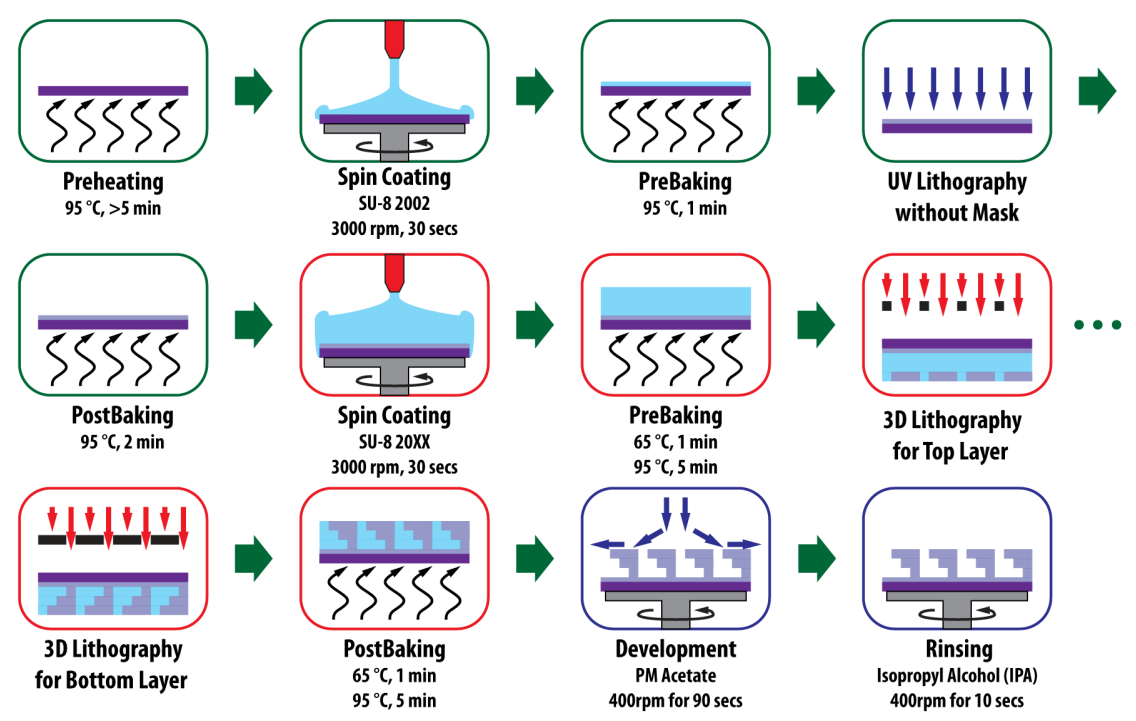

Figure 2.Pictorial description of SU-8 Sample preparation process. Standard protocol for SU-8 was used except for lithography process. To improve the adhesion between cover glass and SU-8, $2 \mu \mathrm{m}$ thick SU-8 layer was attached on cover glass surface before coating thick layers.
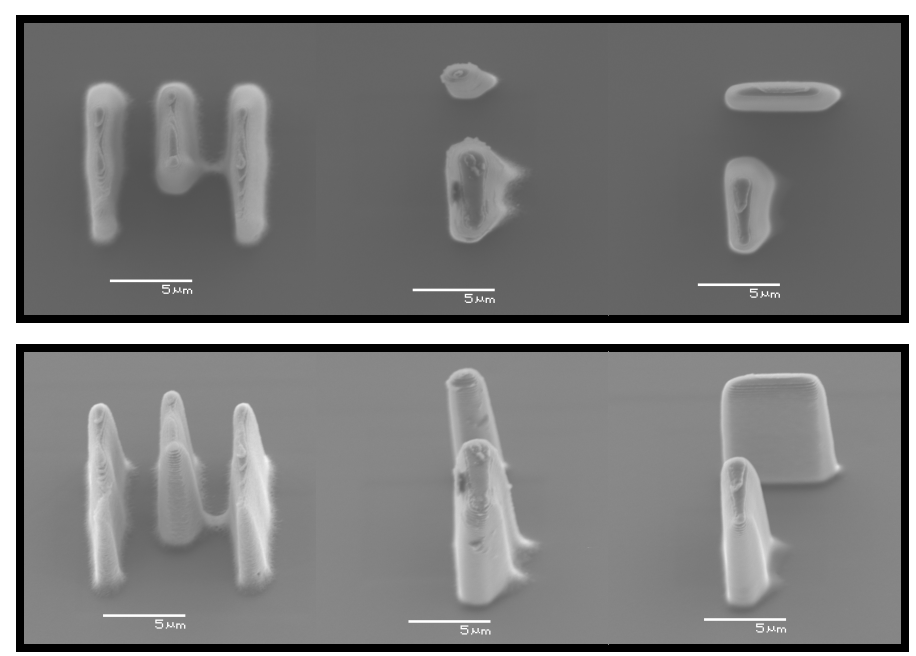

Figure 3. 2.5D structure for MIT logo: top view (top) and isometric view (bottom). The thickness of the SU-8 coating was $15 \mu \mathrm{m}$ and 2D structures are stacked up with the step size of $500 \mathrm{~nm}$. 40X objective with 1.3 NA oil immersion was used. Energy dose per layer was $94.3 \mu \mathrm{J} / \mu \mathrm{m}^{2}$ 


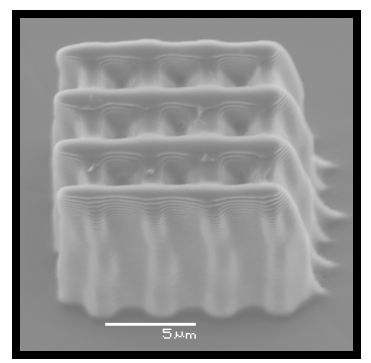

(a)

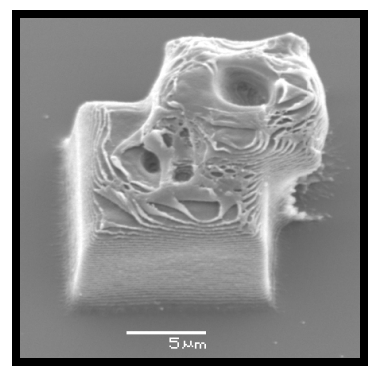

(b)

Figure 4. (a) 3D photonic crystal structure and (b) high-aspect-ratio structures. The thickness of the SU-8 coating was (a) 15 $\mu \mathrm{m}$ and (b) $10 \mathrm{um}$. 2D structures are stacked up with the step size of (a) $500 \mathrm{~nm}$ and (b) $200 \mathrm{~nm}$. $40 \mathrm{X}$ objective with $1.3 \mathrm{NA}$ oil immersion was used. Energy dose per layer was (a) $188.7 \mu \mathrm{J} / \mu \mathrm{m}^{2}$ and (b) $11.8 \mu \mathrm{J} / \mu \mathrm{m}^{2}$

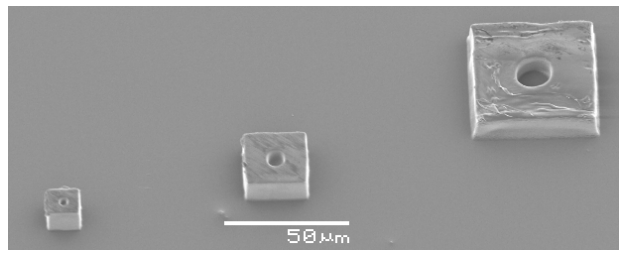

Figure 5. Different scale structures with various objectives. Note that same optical mask was used for all the structure fabrication. Different objectives were used: from left to right, 40X (1.3NA, oil immersion), 20X(0.75NA, air), and 10X (0.5NA, air). The thickness of the SU-8 coating was $15 \mu \mathrm{m}$ and 2D structures are stacked up with the step size of $500 \mathrm{~nm}$. Energy dose per layer was $188.7 \mu \mathrm{J} / \mu \mathrm{m}^{2}$

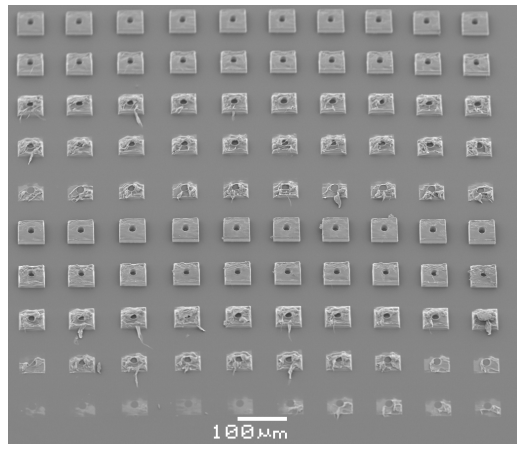

Figure $6.1 \mathrm{~mm}$ by $1 \mathrm{~mm}$ large scale structures with translating sample stage (Stitching). The thickness of the SU-8 coating was $10 \mu \mathrm{m}$ and 2D structures are stacked up with the step size of $500 \mathrm{~nm}$. 10X objective with $0.5 \mathrm{NA}$ air was used. The structures in same row are identical. Each row has different exposure time, or energy dose: from top to bottom, 188.7 $\mu \mathrm{J} / \mu \mathrm{m}^{2}, 94.3 \mu \mathrm{J} / \mu \mathrm{m}^{2}, 37.7 \mu \mathrm{J} / \mu \mathrm{m}^{2}, 18.9 \mu \mathrm{J} / \mu \mathrm{m}^{2}$, and $9.4 \mu \mathrm{J} / \mu \mathrm{m}^{2}$

\section{SUMMARY}

In summary, we introduced and implemented 3D lithographic microfabrication system based on temporal focusing concept. With our 3D microfabrication technique, depth-resolving fabrication, high-aspect-ratio structure, scalable capability, high-throughput production were demonstrated. Assuming that 3D lithographic microfabrication is possible, we expect many high-throughput applications in the biomedical field since one of its advantages is scalability. Microfluidic device has been very popular and has the potential to deliver very complex biomedical analysis. However, 3D microfluidic device fabrication is currently confined to laboratory setting since there are very few methods for mass 
production. Another potential application is cell patterning in tissue scaffold, which is mostly done in 2D. Therefore, 3D lithographic microfabrication has mass production potential for many biomedical applications.

\section{ACKNOWLEDGEMENT}

This work is supported by MIT Deshphande Center for Technological Innovation, Singapore-MIT Alliance (SMA), and Singapore-MIT Alliance for Research and Technology (SMART).

\section{REFERENCES}

[1] J. H. Strickler, and W. W. Webb, “3-Dimensional Optical-Data Storage in Refractive Media by 2-Photon Point Excitation," Optics Letters, 16(22), 1780-1782 (1991).

[2] D. Yang, S. J. Jhaveri, and C. K. Ober, "Three-dimensional microfabrication by two-photon lithography," Mrs Bulletin, 30(12), 976-982 (2005).

[3] S. Maruo, and J. T. Fourkas, "Recent progress in multiphoton microfabrication," Laser \& Photonics Reviews, 2(1-2), 100-111 (2008).

[4] C. E. Olson, M. J. R. Previte, and J. T. Fourkas, "Efficient and robust multiphoton data storage in molecular glasses and highly crosslinked polymers," Nature Materials, 1(4), 225-228 (2002).

[5] B. H. Cumpston, S. P. Ananthavel, S. Barlow et al., "Two-photon polymerization initiators for threedimensional optical data storage and microfabrication," Nature, 398(6722), 51-54 (1999).

[6] G. D. Pins, K. A. Bush, L. P. Cunningham et al., "Multiphoton excited fabricated nano and micro patterned extracellular matrix proteins direct cellular morphology," Journal of Biomedical Materials Research Part A, 78A(1), 194-204 (2006).

[7] F. Claeyssens, E. A. Hasan, A. Gaidukeviciute et al., "Three-Dimensional Biodegradable Structures Fabricated by Two-Photon Polymerization," Langmuir, 25(5), 3219-3223 (2009).

[8] M. Straub, M. Ventura, and M. Gu, "Multiple higher-order stop gaps in infrared polymer photonic crystals," Physical Review Letters, 91(4), - (2003).

[9] R. Guo, Z. Y. Li, Z. W. Jiang et al., "Log-pile photonic crystal fabricated by two-photon photopolymerization," Journal of Optics a-Pure and Applied Optics, 7(8), 396-399 (2005).

[10] J. Wu, D. Day, and M. Gu, "A microfluidic refractive index sensor based on an integrated three-dimensional photonic crystal," Applied Physics Letters, 92(7), - (2008).

[11] M. Stoneman, M. Fox, C. Y. Zeng et al., "Real-time monitoring of two-photon photopolymerization for use in fabrication of microfluidic devices," Lab on a Chip, 9(6), 819-827 (2009).

[12] D. Oron, E. Tal, and Y. Silberberg, "Scanningless depth-resolved microscopy," Optics Express, 13(5), 14681476 (2005).

[13] G. H. Zhu, J. van Howe, M. Durst et al., "Simultaneous spatial and temporal focusing of femtosecond pulses," Optics Express, 13(6), 2153-2159 (2005).

[14] D. Oron, and Y. Silberberg, "Harmonic generation with temporally focused ultrashort pulses," Journal of the Optical Society of America B-Optical Physics, 22(12), 2660-2663 (2005).

[15] M. E. Durst, G. H. Zhu, and C. Xu, "Simultaneous spatial and temporal focusing for axial scanning," Optics Express, 14(25), 12243-12254 (2006).

[16] H. Suchowski, D. Oron, and Y. Silberberg, "Generation of a dark nonlinear focus by spatio-temporal coherent control," Optics Communications, 264(2), 482-487 (2006).

[17] M. E. Durst, G. Zhu, and C. Xu, "Simultaneous spatial and temporal focusing in nonlinear microscopy," Optics Communications, 281(7), 1796-1805 (2008).

[18] W. H. Teh, U. Durig, G. Salis et al., "SU-8 for real three-dimensional subdiffraction-limit two-photon microfabrication," Applied Physics Letters, 84(20), 4095-4097 (2004). 